\title{
Infecção do Anopheles (Kerteszia) cruzii por Plasmodium vivax e Plasmodium vivax variante VK247 nos Municípios de São Vicente e Juquitiba, São Paulo
}

\author{
Maria Stela Branquinho, ${ }^{1}$ Mauro Toledo Marrelli, ${ }^{2}$ Izilda Curado, ${ }^{2}$ \\ Délsio Natal, ${ }^{3}$ José Maria Soares Barata, ${ }^{3}$ Rosa Tubaki, ${ }^{1}$ Glória Cristina \\ Carréri-Bruno, ${ }^{1}$ Regiane Tironi de Menezes ${ }^{1}$ e Judith K. Kloetze/2,4
}
RESUMO O Estado de São Paulo, situado na região Sudeste do Brasil, apresenta esporadicamente casos autóctones de malária que se caracterizam pela presença de quadro clínico benigno com para- sitemias baixas e sintomatologia branda, identificados como malária vivax. Pouco se sabe a respeito da sintomatologia e resposta imune desenvolvidas pelo ser humano para as variantes Plasmodium vivax VK247 e Plasmodium vivax-like humano. Estas variantes são transmi- tidas pelo mosquito Anopheles (Kerteszia) cruzii, uma das espécies mais abundantes no Sudeste brasileiro. O objetivo deste trabalho foi verificar a infecção em anofelinos desta região, através do teste imunoenzimático ELISA com utilização de anticorpos monoclonais específicos dirigidos contra as regiões repetitivas da proteina circunsporozoíta de P. vivax clássico, P. brasilianum/P. malariae $e$ P. vivax VK247. Coletas entomológicas foram realizadas no período de 1991 a 1993 em São Vicente e Juquitiba, municípios localizados em área remanes- cente da Mata Atlântica do Estado de São Paulo. A Mata Atlântica é rica em plantas da famí- lia Bromeliaceae, criadouros de formas imaturas de anofelinos do subgênero Kerteszia. De um total de 1117 espécimes de An. (Ker.) cruzii capturados no Município de São Vicente, 0,179\% foram positivos para P. vivax clássico. Em Juquitiba, dentre 1161 An. (Ker.) cruzii pesqui- sados, 0,086\% foram positivos para o $\mathrm{P}$. vivax VK247, o que demonstra a presença da variante na região. Embora o indice de infecção encontrado seja baixo, a alta densidade destes mosqui- tos e sua voracidade (picam durante as $24 \mathrm{~h}$ do dia) poderiam compensar a baixa porcentagem de espécimes infectados.

A malária é doença de elevada prevalência em áreas tropicais e subtropi-

1 Superintendência de Controle de Endemias (SUCEN). Correspondência e pedidos de separatas devem ser enviados a Maria Stela Branquinho no seguinte endereço: Rua Cardeal Arcoverde, 2878, CEP 05408-000, São Paulo, SP, Brasil.

2 Universidade de São Paulo, Instituto de Medicina Tropical, São Paulo, SP, Brasil.

3 Universidade de São Paulo, Faculdade de Saúde Pública, São Paulo, SP, Brasil.

4 Universidade de São Paulo, Instituto de Ciências Biomédicas, São Paulo, SP, Brasil. cais do mundo e representa um obstáculo para o desenvolvimento sócioeconômico dos países afetados. O Brasil é responsável por $47 \%$ dos casos de malária registrados nas Américas (1). Em 1993 foram notificados 484135 casos na região Amazônica, sendo que $63,43 \%$ deles foram causados pelo parasita Plasmodium vivax; 35,79\% pelo Plasmodium falciparum; $0,04 \%$ pelo Plasmodium malariae; e $0,74 \%$ pela associação de $P$. vivax e $P$. falciparum (2).
A superfície do estágio de plasmódios é recoberta por uma proteína denominada circunsporozoíta (proteína CS), que apresenta epítopos específicos para cada espécie de plasmódio, representados por seqüências repetitivas de aminoácidos. Tais seqüências são conservadas para o $P$. falciparum, $P$. malariae e $P$. brasilianum. Contudo, para o $P$. vivax clássico, foram descritas variantes desta proteína, denominadas $P$. vivax VK247 e P. vivax-like humano $(3,4)$. 
No Brasil, a variante VK247 foi observada no Estado do Acre, através de inquérito sorológico (5) e através de pesquisa do antígeno CS deste plasmódio em anofelinos (6). No Estado do Pará, as variantes VK247 e vivax-like foram encontradas pela técnica de polymerase chain reaction (PCR) em infecções mistas (7). Cochrane et al. (8) e Lal (9) demonstraram a existência de identidade entre as proteínas CS de P. malariae e P. brasilianum. Qari et al. $(4,10)$ demonstraram a existência de identidade entre as proteínas CS de $P$. vivax-like humano e $P$. simiovale (parasita de malária simiana). Pouco se sabe a respeito da sintomatologia e resposta imune desenvolvidas pelo ser humano em relação às variantes de $P$. vivax, ou mesmo sobre os vetores mais competentes para sua transmissão. Contudo, é possível que exista uma correlação entre casos assintomáticos em áreas malarígenas e presença de variantes.

O Estado de São Paulo é considerado área sem transmissão ativa da doença, porém a Superintendência de Controle de Endemias (SUCEN), órgão da Secretaria de Estado da Saúde, tem detectado anualmente casos esporádicos de malária autóctone, de decurso clínico geralmente atípico, brando ou mesmo assintomático. O parasita identificado nesses casos é o $P$. vivax $(11,12)$. Em 1994 foram notificados 28 casos autóctones no Estado de São Paulo (4,3\% do total de casos notificados). Nos últimos 3 anos, cerca de $90 \%$ dos casos autóctones ocorreram nas regiões da grande São Paulo (Município de Juquitiba), Vale do Ribeira, Baixada Santista e Litoral Norte (13).

Os vetores incriminados na transmissão da malária nestas localidades são o Anopheles (Kerteszia) cruzii e o Anopheles (Kerteszia) bellator (14). São espécies neotropicais, caracterizadas por ampla distribuição ao longo da Costa Atlântica brasileira. Estes anofelinos são conhecidos transmissores de $P$. vivax, bem como de $P$. simium e de $P$. brasilianum (parasitas de malária simiana). O An. (Ker.) cruzii é um anofelino antropofílico de hábitos predominantemente exofílicos, encontrado em ambientes de mata. Apresenta atividade ao longo das 24 horas do dia, com picos nos períodos crepusculares. As formas imaturas se desenvolvem em águas depositadas nos áxeis das bromeliáceas (14-16). A mata da Costa Atlântica abriga espécies de macacos comumente infectados com $P$. simium e $P$. brasilianum $(17,18)$. É possível que estes parasitas sejam transmitidas para o ser humano, o que poderia explicar a sintomatologia escassa.

Os dados sobre a infecção do $A n$. (Ker.) cruzii por plasmódios ainda são restritos. Somente a partir do desenvolvimento de anticorpos monoclonais específicos contra os peptídeos sintéticos correspondentes às regiões repetitivas da proteína CS de diferentes espécies de plasmódios, tornou-se possível a realização de testes imunoenzimáticos e imunorradiométricos para detectar infecção em anofelinos. Essa detecção só era viável anteriormente através da dissecção de glândulas salivares. Os novos testes permitem identificar a espécie de parasita da malária com que um anofelino está infectado, o que não acontece com a visualização do esporozoíta, além de apresentarem alta sensibilidade, especificidade e reprodutibilidade dos resultados. Estes testes são realizados com maior rapidez do que a dissecção de glândulas salivares e possibilitam o processamento dos espécimes conservados para posterior processamento em locais que apresentem infra-estrutura laboratorial adequada. Desta forma, o emprego destas técnicas é importante para o entendimento dos mecanismos de transmissão e para a atualização das estratégias de controle da malária.

O estudo descrito no presente trabalho foi desenvolvido nos Municípios de São Vicente e Juquitiba, Estado de São Paulo, durante o período de 1991 a 1993. O objetivo do estudo foi verificar a infecção de anofelinos, através do teste imunoenzimático ELISA de captura com anticorpos monoclonais dirigidos contra as regiões repetitivas da proteína CS de $P$. vivax clássico, $P$. vivax VK247 e $P$. malariae/P. brasilianum.

\section{MATERIAIS E MÉTODOS}

\section{Área de estudo}

As coletas entomológicas foram realizadas em duas localidades situadas em área remanescente de Mata Atlântica no Estado de São Paulo, cuja vegetação do tipo perenifólia higrófila é rica em plantas da família Bromeliaceae, que funcionam como criadouros de formas imaturas de anofelinos do subgênero Kerteszia. Uma das áreas está inserida na região do Planalto Atlântico a $800 \mathrm{~m}$ de altitude, na Reserva Estadual Pedro de Toledo $\left(24^{\circ} 04^{\prime} \mathrm{S} ; 47^{\circ} 00^{\prime} \mathrm{O}\right)$, Município de Juquitiba, e se limita ao sul com os municípios de Itanhaém e Pedro de Toledo. A outra área localiza-se na encosta serrana, a $600 \mathrm{~m}$ de altitude, no Parque Estadual da Serra do Mar (235' S; 46 $23^{\prime} \mathrm{O}$ ), nas localidades de Pai Mathias e Engenheiro Ferraz, pertencentes ao Município de São Vicente. A região planaltina apresenta índice pluviométrico anual de 1200 a $1400 \mathrm{~mm}$ e temperatura médial anual de 18 a $19{ }^{\circ} \mathrm{C}$. A área serrana exibe média anual de $2000 \mathrm{~mm}$ de chuva e temperatura média de 18 a $22{ }^{\circ} \mathrm{C}(19)$.

\section{Coleta e identificação dos anofelinos}

$\mathrm{Na}$ área de planalto, os mosquitos foram capturados com as técnicas de isca humana com luz e armadilha de Shannon nos hábitats de mata e aberto (das 17 às $20 \mathrm{~h}$ ) e armadilha de Shannon no ambiente peridomiciliar (das 17 às 20 h). Os hábitats aberto e mata distam respectivamente 50 e $100 \mathrm{~m}$ do domicílio. $\mathrm{Na}$ área da encosta da serra, procedeu-se às capturas com armadilha de Shannon no ambiente peridomiciliar, a $30 \mathrm{~m}$ da mata (das 17 às $20 \mathrm{~h}$ ). A identificação dos anofelinos foi realizada com base em Forattini (20) e Zavortink (21) e por comparações com material da coleção entomológica da Faculdade de Saúde Pública da Universidade de São Paulo. 


\section{Teste imunoenzimático ELISA}

Foram utilizados anticorpos monoclonais dirigidos contra as regiões repetitivas específicas da proteína CS do $P$. vivax clássico (2F2) (22); P. vivax VK247 (2E10) (3); e P. malariae/P. brasilianum (6B10) (23). Como controles positivos foram utilizados esporozoítas de $P$. vivax clássico (24). Como controles negativos empregou-se anofelinos criados em insetário, sabidamente não infectados por plasmódios.

Após a identificação, os anofelinos fêmeas foram acondicionados em tubos com sílica gel e mantidos em temperatura ambiente até serem processados. Em seguida foram triturados individualmente em $50 \mu \mathrm{l}$ de solução salina tamponada com fosfato (PBS), pH 7,2, contendo albumina bovina (BSA) a $1 \%$; Nonidet P40 $(0,5 \%)$; e os inibidores de protease antipaína e leupeptina $(25 \mu \mathrm{g} / \mathrm{ml})$ e aprotinina (1,7 unidades/ $/ \mu \mathrm{l})$. A cada tubo acrescentou-se $150 \mu \mathrm{l}$ de solução de PBS pH 7,2 contendo BSA $(0,25 \%)$. O material foi mantido a $-20{ }^{\circ} \mathrm{C}$. Os testes foram realizados como descrito anteriormente (5). Placas plásticas de PVC (Dynatech Laboratories, Chantilly, Virgínia, EUA) foram sensibilizadas por $15 \mathrm{~h}$ a $4{ }^{\circ} \mathrm{C}$ com $60 \mu \mathrm{l}$ de anticorpo monoclonal $(1 \mu \mathrm{g} / \mathrm{ml})$ em PBS $(\mathrm{pH} 7,2)$. Após cinco lavagens com solução de PBS $(\mathrm{pH} 7,2)$ contendo 0,05\% de Tween 20 (Sigma) (PBS-T20), as placas foram bloqueadas com 150 $\mu \mathrm{l}$ de PBS-T20, BSA 5\%, permanecendo por $2 \mathrm{~h}$ a $37^{\circ} \mathrm{C}$. Após novo ciclo de lavagens com PBS-T20, foram adicionados $30 \mu \mathrm{l}$ da suspensão de mosquitos previamente triturados, com nova incubação a $37^{\circ} \mathrm{C}$ por $1 \mathrm{~h}$. Em seguida as placas foram submetidas a um novo ciclo de lavagens; $50 \mu \mathrm{l}$ do anticorpo monoclonal biotinilado e previamente titulado foram adicionados, permanecendo por $2 \mathrm{~h}$ a $37^{\circ} \mathrm{C}$. Para cada reação foram utilizados controles positivos e negativos. O mesmo procedimento quanto às lavagens foi novamente realizado, adicionando-se, em seguida, $50 \mu l$ de conjugado peroxidase ligado à estreptoavidina diluído em PBS-T20, BSA 1\%. Após 30 min de incubação a $37{ }^{\circ} \mathrm{C}$, as placas foram lavadas três vezes em solução de PBST20 e três vezes em água destilada, adicionando-se $60 \mu \mathrm{l}$ da mistura cromógena 2,2'azino-di(3-etil-benzatiazoline sulfonato) (Kirkegaard \& Perry Laboratory, Gaithersburg, Maryland, EUA). Após $30 \mathrm{~min}$ em temperatura ambiente, a reação foi interrompida pela adição de $50 \mu \mathrm{l}$ de dodecil sulfato de sódio (SDS) a $20 \%$ em água destilada. Os valores das densidades ópticas foram obtidos após leitura das reações a $414 \mathrm{~nm}$ em leitor de ELISA (MULTISKAN MCC/340P).

O limiar de reatividade, ou cut-off (CO), do teste imunoenzimático foi definido para cada uma das espécies de plasmódios estudadas, a partir de 100 fêmeas de An. stephensi criadas em laboratório, não infectadas, adicionando-se 3 desvios padrão à média aritmética das densidades ópticas obtidas em diferentes ensaios, segundo a fórmula $\mathrm{CO}=\mathrm{X}+3 \mathrm{SD}$ (onde $\mathrm{X}$ é a média aritmética das densidades ópticas dos espécimes padrão negativos e SD é o desvio padrão obtido). Os valores das densidades ópticas dos exemplares positivos foram classificados em cruzes, sendo uma cruz (+) de 3 a 6 desvios padrão; duas cruzes $(++)$ de 7 a 9 desvios padrão; e três cruzes $(+++)$ mais de 9 desvios padrão (6).

\section{RESULTADOS}

Um total de 2278 Anopheles (Kerteszia) cruzii foram analisados pelo teste ELISA, capturados no Município de São Vicente, nas localidades de Pai
Mathias (517) e Engenheiro Ferraz (600), e no Município de Juquitiba (1161). Dos 1117 mosquitos capturados nas duas localidades de São Vicente, dois exemplares $(0,179 \%)$ foram positivos para o $P$. vivax clássico. Os demais anofelinos coletados neste município foram negativos para o P. vivax VK247 e para o P. brasilianum/malariae. No município de Juquitiba, dentre os 1161 mosquitos examinados, um exemplar $(0,086 \%)$ foi positivo para o $P$. vivax VK247, demonstrando assim a presença desta variante na região estudada. Os outros exemplares foram negativos para as demais espécies de plasmódios pesquisadas.

As médias aritméticas das densidades ópticas para o $P$. vivax, para o $P$. vivax VK247 e para o P. malariae/brasilianum foram, respectivamente, 0,152 ; 0,182 ; e 0,206 , com desvio padrão de 0,$024 ; 0,046 ;$ e 0,039 . Os valores de cutoff obtidos foram 0,$225 ; 0,322$; e 0,323 , respectivamente.

Os valores de densidades ópticas obtidos com os mosquitos positivos foram de 1,412 para o $P$. vivax clássico e 1,465 para o $P$. vivax VK 247. Segundo os critérios referidos anteriormente, tais mosquitos foram classificados em +++ (valores maiores do que 9 desvios padrão em relação ao limiar de reatividade).

Na tabela 1 observa-se a ocorrência de anofelinos segundo técnica de captura empregada. $\mathrm{O}$ maior rendimento das coletas foi obtido através da utilização de armadilha de Shannon nas três localidades estudadas. Os exemplares positivos para $P$. vivax e $P$. vivax

TABELA 1. Ocorrência de Anopheles (Kerteszia) cruzii segundo técnica de captura empregada nas localidades de Pai Mathias, Engenheiro Ferraz e Juquitiba, SP, Brasil, 1991 a 1993

\begin{tabular}{lccc}
\hline & \multicolumn{2}{c}{ Técnica de captura } & \\
\cline { 2 - 3 } \multicolumn{1}{c}{ Localidades } & Isca humana & Shannon & Total \\
\hline Engenheiro Ferraz & 192 & 408 & 600 \\
Pai Mathias & 203 & 314 & 517 \\
Juquitiba & 65 & 1096 & 1161 \\
$\quad$ Total & 460 & 1818 & 2278 \\
\hline
\end{tabular}


VK247 foram obtidos através de armadilha de Shannon.

\section{DISCUSSÃO}

Embora o índice de infecção dos anofelinos obtido neste trabalho seja aparentemente baixo $(0,149 \%$ para o $P$. vivax clássico e $0,086 \%$ para o $P$. vivax VK247), foi suficiente para que três funcionários da SUCEN contraíssem malária durante as coletas realizadas em Pai Mathias e Engenheiro Ferraz (25). An. (Ker.) cruzii são mosquitos vorazes que picam durante as 24 horas do dia (com picos crepusculares). Além disso, nos meses em que normalmente são notificados os casos de malária na região (meses com temperaturas mais elevadas), a densidade desses anofelinos é extremamente alta. Estes fatores poderiam compensar a baixa porcentagem de espécimes infectados.

Zavala et al. (26) descreveram a associação entre carga parasitária e valores de densidade óptica com potencialidade vetorial, que foi utilizada no presente estudo para determinação do limiar de reatividade e da carga parasitária dos exemplares coletados. Observando nossos resultados, verificamos que os valores das densidades ópticas dos exemplares positivos foram muito mais elevados do que os limiares de reatividade, o que indica uma elevada carga parasitária nestes anofelinos.

A presença de anofelinos infectados com $P$. vivax clássico e com a variante $P$. vivax VK247 sugere a possibilidade de uma diversidade de plasmódios circulando nesta região. Os dados de sorologia da população residente (27) reforçam esta sugestão.

Curado et al. (28) realizaram inquéritos sorológicos nas mesmas regiões e época deste trabalho. Estes autores empregaram os testes imunoenzimático (ELISA) com peptídeos sintéticos como antígenos específicos das proteínas CS de $P$. malariae/P. brasilianum, $P$. vivax clássico, $P$. vivax VK247 e $P$. vivax-like humano, além da reação de imunofluorescência indireta (IFI) com antígeno de formas sangüíneas assexuadas de $P$. vivax. As prevalências totais obtidas através do teste de ELISA em 475 amostras de soro coletadas em um raio de 5 a $10 \mathrm{~km}$ em torno da moradia dos casos de malária autóctone notificados foram: 3,6\% para o P. malariae/P. brasilianum; 5,3\% para $P$. vivax-like humano; $7,1 \%$ para $P$. vivax VK247 e $4,3 \%$ para o $P$. vivax clássico, sendo de $29,9 \%$ a positividade em IFI, reforçando os dados do presente estudo. Os resultados apresentados por Curado et al. $(27,28)$ sugerem uma subnotificação dos casos de malária, principalmente os causados por espécies de plasmódios como $P$. malariae/P. brasilianum, ocasionada provavelmente pela baixa sensibilidade aliada à dificuldade da identificação do parasita no exame microscópico direto.

O Estado de São Paulo apresenta casos autóctones de malária cuja etiologia continua obscura. É importante conhecer a extensão do problema e verificar se estes casos se tratam de transmissão silvestre que acomete o homem acidentalmente.
O comportamento e distribuição do An. (Ker.) cruzii foram discutidos em detalhe por diversos autores (14-16). Como afirmamos anteriormente, porém, os dados sobre a infecção destes mosquitos por plasmódios ainda são restritos. $\mathrm{O}$ emprego das técnicas utilizadas no presente estudo é importante para o entendimento dos mecanismos de transmissão e para a atualização das estratégias de controle da malária. Além disso, a possibilidade da existência de malária assintomática no Brasil $(29,30)$ e de uma correlação entre casos assintomáticos em áreas malarígenas e presença de variantes de $P$. vivax reforça a importância de estudos que abordem aspectos clínicos, de prevalência de anticorpos antiCS de variantes deste plasmódio, para o monitoramento da transmissão e emprego de eventuais vacinas que possam surgir nos próximos anos. Também são importantes estudos entomológicos (determinação das espécies vetoras e do índice de infecção por plasmódios) e de resistência a antimaláricos.

Agradecimentos. Agradecemos à Dra. Ruth Nussenzweig, New York University, pelos anticorpos monoclonais, proteínas recombinantes e controles negativos dos testes; e à SUCEN pela infra-estrutura de campo. Auxílio financeiro dos Laboratórios de Investigações Médicas (LIM 47) da Faculdade de Medicina da Universidade de São Paulo.

\section{REFERÊNCIAS}

1. World Health Organization (WHO). World malaria situation in 1993. Weekly Epidemiol Rec 1996;71:17-48.

2. Brasil, Ministério da Saúde, Fundação Nacional de Saúde, Gerência Técnica de Malária. Dados epidemiológicos de malária, 1993. Brasília: Fundação Nacional de Saúde, Gerência Técnica de Malária; 1994.

3. Rosenberg R, Wirtz RA, Lanar DE, Sattabongkot J, Hall T, Waters AP, Prasittisuk C.
Circumsporozoite protein heterogeneity in the human malaria parasite Plasmodium vivax. Science 1989;245:973-976.

4. Qari SH, Shi Y, Goldman TF, Udhayakumar $\mathrm{V}$, Alpers MP, Collins WE, et al. Identification of Plasmodium vivax-like human malaria parasite. Lancet 1993;341:780-783.

5. Kremsner PG, Neifer S, Zotter GM, Bienzle U, Rocha RM, Maracic M, et al. Prevalence and level of antibodies to the circumsporozoite proteins of human malaria parasites including a variant of Plasmodium vivax, in the population of two epidemiologically distinct areas in the State of Acre, Brazil. Trans $R$ Soc Trop Med Hyg 1992;86;23-27.

6. Branquinho MS, Taipe-Lagos CB, Rocha R, Natal D, Barata JMS, Cochrane AH, et al. Anophelines in the State of Acre, Brazil, infected with Plasmodium falciparum, Plasmodium vivax, the variant Plasmodium vivax VK247 
and P. malariae. Trans $R$ Soc Trop Med Hyg 1993;87:391-394.

7. Oliveira D, Qari S, Machado RLD, Oliveira S, Póvoa M, Collins W, et al. Prevalence of $P$. vivax-like human parasite in a malaria endemic region of Brazil. Rev Patol Trop 1995; 23:236.

8. Cochrane AH, Collins WE, Nussenzweig RS. Monoclonal antibody identifies circumsporozoite protein of Plasmodium malariae and detects common epitope on Plasmodium brasilianum sporozoites. Infect Immun 1984;45: 592-595.

9. Lal AA. Structure of the circumsporozoite gene of Plasmodium malariae. Mol Biochem Parasitol 1988;30:291-294.

10. Qari SH, Shi Y, Povoa MM, Alpers MP, Deloron F, Murphy GS, et al. Global occurrence of Plasmodium vivax-like human malaria parasite. I Infect Dis 1993;168: 1485-1489.

11. Carvalho ME, Glasser CM, Ciaravolo RMC, Etzel A, Santos LA, Ferreira CS. Sorologia de malária vivax no foco Aldeia dos Índios, Município de Peruíbe, São Paulo, 1984 a 1986. Cad Saude Publ Rio de Janeiro 1988;4: 276-292

12. Carvalho ME, Glasser CM, Santo LA, Ciaravolo RMC. Nota sobre o encontro de casos autóctones de malária vivax por meio de técnica sorológica em São Paulo. Cad Saude Publ Rio de Janeiro 1985;1:250-252.

13. Secretaria de Estado da Saúde do Estado de São Paulo, Superintendência do Controle de Endemias (SUCEN). Relatório de Avaliação do Programa de Controle de Malária. São Paulo: SUCEN; 1994.

14. Forattini OP, Gomes AC, Natal D, Kakitani I, Marucci D. Preferências alimentares de mosquitos Culicidae no Vale do Ribeira, São Paulo, Brasil. Rev Saude Publ 1987;21:171-187.

15. Gadelha P. From "forest malaria" to "bromeliad malaria": a case-study of scientific controversy and malaria control. Parasitologia 1994;36:175-195.

16. Tubaki RM. Biting activity of Anopheles (Kerteszia) cruzii (Culicidae) in domiciliar habitats in the Southern Atlantic Forest Peruíbe, State of São Paulo, Brazil. Rev Bras Entomol 1993;37:559-575.

17. Deane LM, Ferreira Neto JA, Lima MM. The vertical dispersion of Anopheles (Kerteszia) cruzii in a forest in Southern Brazil suggests that human cases of malaria of simium origin might be expected. Mem Inst Oswaldo Cruz 1984;79:461-463.

18. Deane LM. Simian malaria in Brazil. Mem Inst Oswaldo Cruz 1992;79:1-120.

19. Troppmair H. Regiões ecológicas do Estado de São Paulo. São Paulo: Universidade de São Paulo; 1975:1-24. (Série biogeografia no. 10).

20. Forattini OP. Entomologia médica. Vol 1. São Paulo: Editora da USP; 1965:474-481.

21. Zavortink TJ. Mosquitoes studies (Diptera, Culicidae) XXIX: A review of the subgenus Kerteszia of Anopheles. Contrib Am Entomol Inst 1973;9:1-54.

22. Nardin EH, Nussenzweig RS, Nussenzweig V, Harinasuta KT, Collins WE, Tapchaisri P et al. Circumsporozoite (CS) proteins of human malaria parasites $P$. falciparum and $P$. vivax. J Exp Med 1982;156:20.

23. Cochrane AH, Nardin EH, Arruda M, Maracic M, Clavijo P, Collins WE, Nussenzweig RS. Widespread reactivity of human sera with a variant of the circumsporozoite protein of Plasmodium vivax. Am J Trop Med Hyg 1990;43:446-451.

24. Barr PJ, Gibson HL, Enea V, Arnot DE, Hollingdale R, Nussenzweig V. Expression in yeast of a Plasmodium vivax antigen of potential use in human malaria vaccine. J Exp Med 1987;165:1160-1171.

25. Carréri-Bruno GC, Ciaravolo RMC, Pereira M. Malária adquirida durante atividade ento- mológica na Serra do Mar, região Sudeste do Brasil. Rev Saude Publica 1995;29:142-143.

26. Zavala F, Matsuda A, Graves PM, Nussenzweig V, Nussenzweig RS. Ubiquity of the repetitive epitope of the CS protein in different isolates of human malaria parasites. J Immunol 1982;135:2790-2793.

27. Curado I, Duarte AM, Lal AA, Nussenzweig RS, Oliveira DA, Oliveira $S$, et al. Serologic investigation of human Plasmodium vivax-like malaria in several localities in the state of São Paulo. Program and Abstracts of the 44th Annual Meeting of the American Society of Tropical Medicine and Hygiene. Am J Trop Med Hyg 1995;52:238.

28. Curado I, Duarte AMRC, Lal AA, Oliveira SG, Kloetzel JK. Antibodies anti blood stream and circumsporozoite antigens (Plasmodium vivax and Plasmodium malariae/P. brasilianum) in areas of very low malaria endemicity in Brazil. Mem Inst Oswaldo Cruz 1997;92: 235-243.

29. Malafronte RS, Valdivia JL, Nakaie CR, Kloetzel JK. Seasonal variation of anti-RESA/PF155 Plasmodium falciparum antibodies in three localities in the state of Amapa, Brazil. Rev Inst Med Trop Sao Paulo 1994;36:237-243.

30. Silva RSU, Santos ECO, Loureiro ECB, Jesus IM, Souza V, Brabo EES, et al. Malária assintomática - uma das causas da perpetuação da transmissão da malária. IV Reunião Nacional dos Pesquisadores em Malária. 1993;31.

Manuscrito recebido em 18 de dezembro de 1995. Aceito em versão revisada em 31 de maio de 1996

Sporadic cases of autochthonous malaria have been recorded in São Paulo State, located in the Southeast region of Brazil. These cases are characterized by their benign course, low parasitemia, and mild symptomatology and have been identified as vivax malaria. Little is known about the symptoms and immune response elicited in humans by the variants Plasmodium vivax VK247 and P. vivax-like human malaria parasites. These variants are transmitted by Anopheles (Kerteszia) cruzii, one of the most common species of mosquitoes in the Southeast of Brazil. The objective of the study described in this paper was to investigate infection in anophelines using ELISA immunoenzymatic assay with specific monoclonal antibodies directed against the repetitive regions of the circumsporozoite protein in classic $P$. vivax, $P$. brasilianum $/ P$. malariae, and P. vivax VK247. Between 1991 and 1993, mosquitoes were collected in São Vicente and Juquitiba, municipalites located in a remnant of the Brazilian Atlantic forest in São Paulo State, an ecosystem rich in plants of the Bromeliaceae family. These plants function as nurseries for immature forms of anophelines of the subgenus Kerteszia. Of 1117 An. (Ker.) cruzii captured in São Vicente, 0.179\% were positive for classic P. vivax. In Juquitiba, of 1161 An. (Ker.) cruzii, $0.086 \%$ were positive for $P$. vivax VK247, confirming the presence of this variant in the region. Although the infection rate is low, the high density of these mosquitoes and their voracity (they exhibit 24-h biting activity) could compensate for the low percentage of infected specimens. 